\title{
The van Hemmen model and effect of random crystalline anisotropy field
}

\author{
Denes M. de Morais ${ }^{a}$, Mauricio Godoy ${ }^{a, *}$, Alberto S. de Arruda ${ }^{a}$, Jonathas N. da Silva ${ }^{b}$, \\ J. Ricardo de Sousa ${ }^{c}$ \\ a Instituto de Física, Universidade Federal de Mato Grosso, 78060-900 Cuiabá, Mato Grosso, Brazil \\ ${ }^{\mathrm{b}}$ Universidade Estadual Paulista, 14800-901, Araraquara, São Paulo, Brazil \\ ${ }^{\mathrm{c}}$ Instituto Nacional de Sistemas Complexos, Departamento de Fisica, Universidade Federal do Amazona, 69077-000, Manaus, Amazonas, Brazil
}

\section{A R T I C L E I N F O}

\section{Article history:}

Received 29 June 2015

Accepted 10 September 2015

Available online 12 September 2015

Keywords:

Random crystalline field

Van Hemmen model

Phase diagram

\begin{abstract}
A B S T R A C T
In this work, we have presented the generalized phase diagrams of the van Hemmen model for spin $S=1$ in the presence of an anisotropic term of random crystalline field. In order to study the critical behavior of the phase transitions, we employed a mean-field Curie-Weiss approach, which allows calculation of the free energy and the equations of state of the model. The phase diagrams obtained here displayed tricritical behavior, with second-order phase transition lines separated from the first-order phase transition lines by a tricritical point.
\end{abstract}

(c) 2015 Elsevier B.V. All rights reserved.

\section{Introduction}

The interest in understanding the physical properties of disordered systems provides a fertile ground of novel models and studies in statistical physics. Many disordered systems are known to be of fundamental importance, including the so-called spin-glass (SG) [1-3] and the random field Ising model (RFIM). The RFIM is a topic of interest current and one of the reasons for this is its physical realization as a diluted Ising antiferromagnet in the presence of a uniform magnetic field along the uniaxial direction [4]. Several of its properties have been revealed, particularly the lower critical $\left(d_{l}=2\right)$ dimension above which a stable ferromagnetic state exists at low temperatures [5]. The phase diagram is another interesting feature of RFIM. Here, the use of the mean-field theory has shown that the nature of the phase transition depends on the distribution associated with the random magnetic field. For Gaussian distribution, the phase transition is always continuous [6], and with a symmetric bimodal distribution the phase transition is continuous (second-order) in the region of low and high temperatures, becoming discontinuous (first-order) for sufficiently large values of magnetic field (random) and low temperature [7]. The trimodal distribution [8-11] indicates a critical $p_{c}$ for which the tricritical behavior disappears. Other studies have indicated that in the three-dimensional case a jump in magnetization and no latent heat is observed [12,13]. Moreover, also very important systems are the spin glasses [14]. Research in spin glasses began in the 1970s after the discoveries of a peak in AC susceptibility in dilute alloys of gold and

\footnotetext{
* Corresponding author.

E-mail addresses: mgodoy@fisica.ufmt.br (M. Godoy), aarruda@fisica.ufmt.br (A.S. de Arruda).
}

iron [15]. Basically, the spin glasses are dilute magnetic alloys, where the interactions between spins are randomly ferromagnetic or antiferromagnetic distributed, which are examples of frozen disorder. This is a necessary requirement for having a locally random competition between ferromagnetic and antiferromagnetic interactions. The presence of disorder introduces frustration and produces difficulties for the system to form an optimized configuration. These heterogeneities of the couplings lead to frustrated spin arrangements, that is, for any choice of orientations of spins leaves we have at least one frustrated bond. There are a large number of examples of spin glass systems, which include CuMn and AuFe alloys [16]. These systems are known to exhibit non-trivial thermodynamic and dynamic properties, which are different when compared to those observed in ordered systems.

Ising-type Hamiltonians with random bonds between the spins are used to spin glass models. In mean-field models the effects of frustration are enhanced, and the thermodynamic that emerges is in some case novel and surprising. On one hand, when compared to ordinary ferromagnets, which host two pure states present below of the transition temperature, the mean-field spin glasses model exhibit at low temperature a complex structure of infinitely large number of equilibrium states, organized in a hierarchical structure. Formally, there is a mathematical approach that enables us to appropriately deal with the quenched disorder present in the Hamiltonian which allows us to compute the thermodynamic properties, but this is very difficult problem to be treated.

Recently, the Ising spin glass with random field similar to model proposed by Sherrington and Kirkpatrick (SK) [17] has been treated for Gaussian [18] and bimodal [19] distributions. The mean-field spin glass model has often been employed in the study of the dynamic anomalies, which are associated with relaxational phenomena in 
supercooled liquids and real glasses [20]. The aging and breakdown of the fluctuation dissipation theorem are intrinsic features of these systems that are captured by dynamic versions of such models. The dynamic calculations are frequently used, but often solutions are found only numerically [21].

It is worth noting that the problems related to spin glasses remain difficult to treat in statistical mechanics [22-24]. Up to now, only mean-field models are exactly tractable, but they require sophisticated mathematical tools $[25,26]$. Therefore, disorder in spin systems is a permanent source of challenging problems. The spin-glass $(S G)$ state is one of the most interesting examples where disorder can provide new physics properties and responses.

In this present work, we have studied the influence of the random crystalline anisotropy field on the spin glass mean field model introduced by van Hemmen [27-29]. This model is exactly solvable and unlike the Sherrington-Kirkpatrick model [17], its solution does not require the use of replica trick. In spite of being not fully realistic, mean-field models give a first qualitative understanding of the thermodynamic behavior of a system.

The outline of the paper is as follows: in Section 2, we have introduced the model, where obtained an analytical expression for the free-energy and equation of states. In Section 3, we presented and discussed the phase diagrams. Finally, Section 4 is devoted to our conclusions.

\section{Formalism and calculation}

The Hamiltonian of the van Hemmen spin-glass model, with an additional ferromagnetic Ising term and a random crystal field, is given by

$\mathcal{H}=-\frac{J_{0}}{N} \sum_{\langle i, j\rangle} S_{i} S_{j}-\sum_{\langle i, j\rangle} J_{i j} S_{i} S_{j}-H \sum_{i} S_{i}-\sum_{i} D_{i} S_{i}^{2}$,

where $J_{0}>0$ is the direct ferromagnetic coupling between the spins in the nearest-neighbor sites iand $j$, which is fundamental to the existence of a mixed phase. $H$ is an external uniform magnetic field and $J_{i j}$ are random interactions between the nearest-neighbor spins, which is given by

$J_{i j}=\frac{J}{N}\left(\xi_{i} \eta_{j}+\xi_{j} \eta_{i}\right)$.

Here, $\xi_{i}$ and $\eta_{j}$ are independent random variables, obeying the probability distribution $P\left(\xi_{i}, \eta_{j}\right)$

$P(\xi, \eta)=\left\{\frac{1}{2}\left[\delta\left(\xi_{i}-1\right)+\delta\left(\xi_{i}+1\right)\right]\right\}\left\{\frac{1}{2}\left[\delta\left(\eta_{j}-1\right)+\delta\left(\eta_{j}+1\right)\right]\right\}$,

which is expected to mimic the presence of disorder in real spin glasses. Moreover, in Eq. (2.1) $\langle i, j\rangle$ denote the sum over all nearestneighbor pairs of spins on a d-dimensional lattice. In that follows, we have considered $S_{i}>1 / 2$ (due to the presence of $D_{i}$ ) which are spin variables integer or half-integer. The crystalline anisotropy fields (or crystal fields) $D_{i}$ are subject to a bimodal probability distribution

$P\left(D_{j}\right)=p \delta\left(D_{j}\right)+(1-p) \delta\left(D_{j}-D\right)$,

where the first term $p \delta\left(D_{j}\right)$ indicates that a fraction $p$ of the spins are free of the influence of crystalline anisotropy field, and in the second term $(1-p) \delta\left(D_{j}-D\right)$ a fraction $(1-p)$ is subject to the crystalline anisotropy field of intensity $D$. Given a configuration of the random variables, the partition function can be written as

$Z_{N}=\operatorname{Tr} \exp \left[\beta \frac{J_{0}}{N} \sum_{\langle i, j\rangle} S_{i} S_{j}+\beta \frac{J}{N} \sum_{\langle i, j\rangle}\left(\xi_{i} \eta_{j}+\xi_{j} \eta_{i}\right) S_{i} S_{j}+\beta H \sum_{i} S_{i}+\beta \sum_{i} D_{i} S_{i}^{2}\right]$,

where $\beta=1 / k_{B} T$ is the inverse of temperature and $\beta J_{0}=K_{0}$ e $\beta J=K$.
Using the following identities:

$$
\sum_{\langle i, j\rangle}\left(\xi_{i} \eta_{j}+\xi_{j} \eta_{i}\right) S_{i} S_{j}=\frac{1}{2}\left[\left(\sum_{i}\left(\xi_{i}+\eta_{i}\right) S_{i}\right)^{2}-\left(\sum_{i} \xi_{i} S_{i}\right)^{2}-\left(\sum_{i} \eta_{i} S_{i}\right)^{2}-2 \sum_{i} \xi_{i} \eta_{i} S_{i}^{2}\right],
$$

$\sum_{\langle i, j\rangle} S_{i} S_{j}=\frac{1}{2}\left[\left(\sum_{i} S_{i}\right)^{2}-\sum_{i} S_{i}^{2}\right]$

$\exp \left(\lambda a^{2}\right)=\frac{1}{\sqrt{2 \pi}} \int_{-\infty}^{\infty} \exp \left(-\frac{x^{2}}{2}+a x \sqrt{2 \lambda}\right) d x$

and the procedures employed in Refs. [24,23], the free energy functional and the equation of states for the ferromagnetic phase (magnetization) and the spin-glass phase (parameter $q$ ) are given by

$$
\begin{aligned}
\beta f= & p \times\left\{\frac{1}{2}\left[K_{0} m^{2}+2 K q^{2}\right]-\frac{1}{4}\left\{\ln \left[\sum_{m_{s}} \cosh \left[\left|m_{s}\right|\left\{K_{0} m+2 K q+\beta H\right\}\right]\right]\right.\right. \\
& +2 \ln \left[\sum_{m_{s}} \cosh \left[\left|m_{s}\right|\left\{K_{0} m+\beta H\right\}\right]\right] \\
& \left.+\ln \left[\sum_{m_{s}} \cosh \left[\left|m_{s}\right|\left\{K_{0} m-2 K q+\beta H\right\}\right]\right\}\right\} \\
& +(1-p)\left\{\frac{1}{2}\left[K_{0} m^{2}+2 K q^{2}\right]\right. \\
& -\frac{1}{4}\left\{\ln \left[\sum_{m_{s}} \exp \left(m_{s}^{2} \beta D\right) \cosh \left[\left|m_{s}\right|\left\{K_{0} m+2 K q+\beta H\right\}\right]\right]\right. \\
& +2 \ln \left[\sum_{m_{s}} \exp \left(m_{s}^{2} \beta D\right) \cosh \left[\left|m_{s}\right|\left\{K_{0} m+\beta H\right\}\right]\right] \\
& \left.\left.+\ln \left[\sum_{m_{s}} \exp \left(m_{s}^{2} \beta D\right) \cosh \left[\left|m_{s}\right|\left\{K_{0} m-2 K q+\beta H\right\}\right]\right]\right\}\right\}
\end{aligned}
$$

and

$$
\begin{aligned}
m= & \frac{1}{4}\left\{p \left[\frac{\sum_{m_{s}}\left|m_{s}\right| \sinh \left[\left|m_{s}\right|\left\{K_{0} m+2 K q+\beta H\right\}\right]}{\sum_{m_{s}} \cosh \left[\left|m_{s}\right|\left\{K_{0} m+2 K q+\beta H\right\}\right]}\right.\right. \\
& +2 \frac{\sum_{m_{s}}\left|m_{s}\right| \sinh \left[\left|m_{s}\right|\left\{K_{0} m+\beta H\right\}\right]}{\sum_{m_{s}} \cosh \left[\left|m_{s}\right|\left\{K_{0} m+\beta H\right\}\right]} \\
& \left.+\frac{\sum_{m_{s}}\left|m_{s}\right| \sinh \left[\left|m_{s}\right|\left\{K_{0} m-2 K q+\beta H\right\}\right]}{\sum_{m_{s}} \cosh \left[\left|m_{s}\right|\left\{K_{0} m-2 K q+\beta H\right\}\right]}\right] \\
& +(1-p)\left[\frac{\sum_{m_{s}} \exp \left(m_{s}^{2} \beta D\right)\left|m_{s}\right| \sinh \left[\left|m_{s}\right|\left\{K_{0} m+2 K q+\beta H\right\}\right]}{\sum_{m_{s}} \exp \left(m_{s}^{2} \beta D\right) \cosh \left[\left|m_{s}\right|\left\{K_{0} m+2 K q+\beta H\right\}\right]}\right. \\
& +2 \frac{\sum_{m_{s}} \exp \left(m_{s}^{2} \beta D\right)\left|m_{s}\right| \sinh \left[\left|m_{s}\right|\left\{K_{0} m+\beta H\right\}\right]}{\sum_{m_{s}} \exp \left(m_{s}^{2} \beta D\right) \cosh \left[\left|m_{s}\right|\left\{K_{0} m+\beta H\right\}\right]} \\
& \left.+\frac{\sum_{m_{s}} \exp \left(m_{s}^{2} \beta D\right)\left|m_{s}\right| \sinh \left[\left|m_{s}\right|\left\{K_{0} m-2 K q+\beta H\right]\right]}{\sum_{m_{s}} \exp \left(m_{s}^{2} \beta D\right) \cosh \left[\left|m_{s}\right|\left\{K_{0} m-2 K q+\beta H\right\}\right]}\right\},
\end{aligned}
$$




$$
\begin{aligned}
q= & \frac{1}{4}\left\{p \left[\frac{\sum_{m_{s}}\left|m_{s}\right| \sinh \left[\left|m_{s}\right|\left\{K_{0} m+2 K q+\beta H\right\}\right]}{\sum_{m_{s}} \cosh \left[\left|m_{s}\right|\left\{K_{0} m+2 K q+\beta H\right\}\right]}\right.\right. \\
& \left.-\frac{\sum_{m_{s}}\left|m_{s}\right| \sinh \left[\left|m_{s}\right|\left\{K_{0} m-2 K q+\beta H\right\}\right]}{\sum_{m_{s}} \cosh \left[\left|m_{s}\right|\left\{K_{0} m-2 K q+\beta H\right\}\right]}\right] \\
& +(1-p)\left[\frac{\sum_{m_{s}} \exp \left(m_{s}^{2} \beta D\right)\left|m_{s}\right| \sinh \left[\left|m_{s}\right|\left\{K_{0} m+2 K q+\beta H\right\}\right]}{\sum_{m_{s}} \exp \left(m_{s}^{2} \beta D\right) \cosh \left[\left|m_{s}\right|\left\{K_{0} m+2 K q+\beta H\right\}\right]}\right. \\
& \left.\left.-\frac{\sum_{m_{s}} \exp \left(m_{s}^{2} \beta D\right)\left|m_{s}\right| \sinh \left[\left|m_{s}\right|\left\{K_{0} m-2 K q+\beta H\right\}\right]}{\sum_{m_{s}} \exp \left(m_{s}^{2} \beta D\right) \cosh \left[\left|m_{s}\right|\left\{K_{0} m-2 K q+\beta H\right\}\right]}\right]\right\},
\end{aligned}
$$

where

$m_{s}=\sum_{-s}^{s}-s+(-s+1)+\cdots(s-1)+s$.

Thus, we have employed a mean-field Curie-Weiss approach to obtain the three generic (for any spin) equations with random anisotropy, which are the free energy and of the equations of state of the van Hemmen model.

\section{Numerical results}

The phase diagrams of the van Hemmen model is obtained via a detailed numerical analysis of the three equations [free energy (Eq. (2.9)), magnetization (Eq. (2.10)) and the parameter $q$ (Eq. (2.11)], but few properties of the phase diagrams can also be obtained analytically. It is important to notice that the first generalization of the van Hemmen model is an extension of the variables of spins $S=1 / 2$ for $S=1$, and in this context, the crystalline anisotropy field will have a very important role in the topology of the phase diagrams and on the thermodynamic properties of the model. For the case $S=1$, Eqs. (2.9)-(2.11) are reduced, respectively, to

$$
\begin{aligned}
\beta f= & \frac{K_{0} m^{2}}{2}+K q^{2}-\frac{p}{4}\left\{\ln \left[1+2 \cosh \left(K_{0} m+2 K q\right)\right]\right. \\
& \left.+2 \ln \left[1+2 \cosh \left(K_{0} m\right)\right]+\ln \left[1+2 \cosh \left(K_{0} m-2 K q\right)\right]\right\} \\
& -(1-p) \frac{1}{4}\left\{\ln \left[1+2 \exp (\beta D) \cosh \left(K_{0} m+2 K q\right)\right]\right. \\
& +2 \ln \left[1+2 \exp (\beta D) \cosh \left(K_{0} m\right)\right] \\
& \left.+\ln \left[1+2 \exp (\beta D) \cosh \left(K_{0} m-2 K q\right)\right]\right\}
\end{aligned}
$$

$$
\begin{aligned}
m= & \frac{1}{2}\left\{p \left[\frac{\sinh \left(K_{0} m+2 K q\right)}{1+2 \cosh \left(K_{0} m+2 K q\right)}+2 \frac{\sinh \left(K_{0} m\right)}{1+2 \cosh \left(K_{0} m\right)}\right.\right. \\
& \left.+\frac{\sinh \left(K_{0} m-2 K q\right)}{1+2 \cosh \left(K_{0} m-2 K q\right)}\right] \\
& +(1-p)\left[\frac{\exp (\beta D) \sinh \left(K_{0} m+2 K q\right)}{1+2 \exp (\beta D) \cosh \left(K_{0} m+2 K q\right)}\right. \\
& +2 \frac{\exp (\beta D) \sinh \left(K_{0} m\right)}{1+2 \exp (\beta D) \cosh \left(K_{0} m\right)} \\
& \left.\left.+\frac{\exp (\beta D) \sinh \left(K_{0} m-2 K q\right)}{1+2 \exp (\beta D) \cosh \left(K_{0} m-2 K q\right)}\right]\right\},
\end{aligned}
$$

$$
\begin{aligned}
& \text { and } \\
& \begin{aligned}
q= & \frac{1}{2}\left\{p\left[\frac{\sinh \left(K_{0} m+2 K q\right)}{1+2 \cosh \left(K_{0} m+2 K q\right)}-\frac{\sinh \left(K_{0} m-2 K q\right)}{1+2 \cosh \left(K_{0} m-2 K q\right)}\right]\right. \\
& +(1-p)\left[\frac{\exp (\beta D) \sinh \left(K_{0} m+2 K q\right)}{1+2 \exp (\beta D) \cosh \left(K_{0} m+2 K q\right)}\right. \\
& \left.\left.-\frac{\exp (\beta D) \sinh \left(K_{0} m-2 K q\right)}{1+2 \exp (\beta D) \cosh \left(K_{0} m-2 K q\right)}\right]\right\} .
\end{aligned}
\end{aligned}
$$

which are used to construct the full phase diagrams, i.e., to derive all the thermodynamic properties of the system.

Phase transition spin-glass (SG) - paramagnetic $(P)$

In the spin-glass phase the magnetization is zero, thus the expressions of free energy and the spin-glass parameter are reduced to

$$
\begin{aligned}
\beta f_{S G}= & K q^{2}-\frac{p}{2}\{\ln [1+2 \cosh (2 K q)]+\ln 3\} \\
& -\frac{(1-p)}{2}\{\ln [1+2 \exp (\beta D) \cosh (2 K q)]+\ln [1+2 \exp (\beta D)]\},
\end{aligned}
$$$$
q_{S G}=p\left[\frac{\sinh (2 K q)}{1+2 \cosh (2 K q)}\right]+(1-p)\left[\frac{\exp (\beta D) \sinh (2 K q)}{1+2 \exp (\beta D) \cosh (2 K q)}\right] .
$$

Moreover, in the spin-glass to paramagnetic transition the order parameter of the spin-glass phase tends to zero $\left(q_{S G} \rightarrow 0\right)$, and given the fact that in the paramagnetic phase the magnetization is also zero, provide second-order phase transition lines in the phase diagram. Therefore, Eq. (3.4) can be expanded in power series up to first order in $q$, which results in

$e^{\beta D}=\frac{3-2 p K}{6 K-2 p K-6}$.

In the case from the first-order phase transition, the frontiers are obtained by solving the system of equations $(f(S G)=f(P)$ and $q=q(S G)$, where $f_{S G}$ and $f_{P}$ represent the free energy of the spinglass and paramagnetic phases, respectively. The particular case of $p=1$ corresponds to the case where all spins are free from the effect of crystal fields and reproduces the original results obtained by van Hemmen [27], wherein the critical temperature is $T_{c}=2 / 3$. For the case $p=0$, all spins of the lattice are subject to the influence of an anisotropy constant $D$. The numerical analysis of Eq. (3.5) provides the second-order phase transition lines of phase transitions between the spin-glass and paramagnetic phases. Indeed, we will examine two types of phase diagrams, the first for $D<0$ and the second for $D>0$ in the $T / J-D / J$ phase space.

Fig. 1 shows the phase diagram in the $T / J-D / J$ plane of the van Hemmen model with spin $S=1$. It displays the transition between the paramagnetic and spin-glass phases for $D<0$. (i) In the case $p=0$, for small values of $|D|$ and at high-temperatures the phase transition is second-order. When the magnitude of $|D|$ increases, the critical temperature decreases and the second-order phase transition line ends at tricritical point (TCP) located by $(D / J=-0.4650, T / J=0.3160)$. Notice that the first-order phase transition and the critical temperature decreases with increasing at $|D|$, going to zero at $D=-0.5$. The TCP separates the second-order from the first-order phase transitions and the system displays tricritical behavior. (ii) In the range of $0 \leq p \leq 0.18$, the phase diagram shows a region of first-order phase transition at low temperatures. The phase transition is second-order at high temperatures. The two types of phase transitions are separated by tricritical points. (iii) In the case $p>0.18$ the system shows only second-order phase transitions between spin-glass and paramagnetic phases. Taking into account that the anisotropy $D<0$ favors the state 


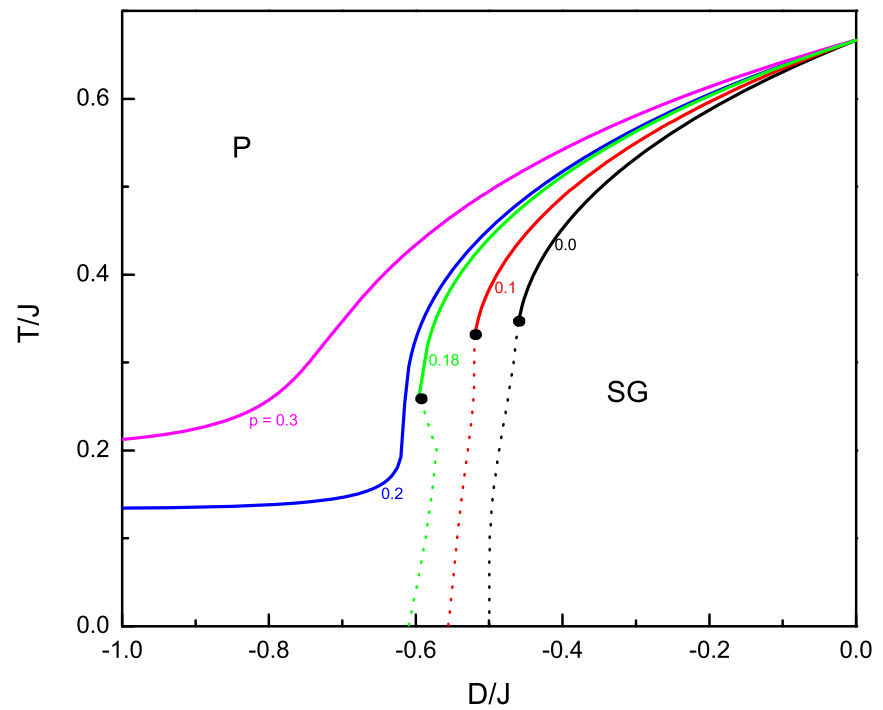

Fig. 1. Phase diagram in the $T / J-D / J$ plane of the van Hemmen model with spin $S=1$ for different values of $p$, as indicated in the figure. The solid and dotted lines represent second- and first-order phase transitions lines, respectively. The black dots represent tricritical points. $P$ and $S G$, denote the paramagnetic and spin-glass phases, respectively.

$S=0$, its enhancement to the negative values leads to a decrease in critical temperature. In this region of the thermodynamic space, the tricritical behavior disappears. Therefore, the increase in the randomness parameter $p$ indicates that a greater amount of spins are free of the influence of the crystalline anisotropy field, indicating that this anisotropy $D$ is essential for the existence of tricritical behavior. The enhancement of the critical temperature is displayed in the phase diagram of Fig. 1. When $p \rightarrow 1$, all the spins are free of the influence of the crystalline anisotropy field. Thus, the behavior of the our model follows the original of van Hemmen model behavior. Furthermore, we also have studied the region with $D>0$. Here, the model does not present tricritical behavior (the phase transition is of second-order type for all values of $p$ ). For second-order phase transition lines, with different values of $p$, starting at $D / J=0$ and $T / J=2 / 3$, the critical temperatures grow with increasing $D / J$. This fact can be understood since positive values of $D$ favors the states $S= \pm 1$, or the spin-glass magnetic order. In the limit $p \rightarrow 1$, we have the pure case with $T_{c}=2 / 3$.

Phase transition: ferromagnetic $(F)$ - paramagnetic $(P)$

Let us now analyze the phase transition between the ferromagnetic and paramagnetic phases $(F-P)$. In the ferromagnetic phase the order parameter $m \neq 0$, whereas the spin-glass parameter is $q=0$, however, in the paramagnetic phase $m=0$ and $q=0$, which simplifies the expressions of free energy and magnetization

$$
\begin{aligned}
\beta f_{F}= & \frac{K_{0} m^{2}}{2}-p\left\{\ln \left[1+2 \cosh \left(K_{0} m\right)\right]\right\}-(1-p) \\
& \left\{\ln \left[1+2 \exp (\beta D) \cosh \left(K_{0} m\right)\right]\right\} .
\end{aligned}
$$

$$
\begin{gathered}
m_{F}=2 p\left[\frac{\sinh \left(K_{0} m\right)}{1+2 \cosh \left(K_{0} m\right)}\right]+2(1-p) \\
{\left[\frac{\exp (\beta D) \sinh \left(K_{0} m\right)}{1+2 \exp (\beta D) \cosh \left(K_{0} m\right)}\right] .}
\end{gathered}
$$

In the phase transition $F-P, m \rightarrow 0$ allows an expansion in power series of Eq. (3.7) as function of $m$, which leads to

$$
e^{\beta D}=\frac{3-2 p K_{0}}{3\left(2 K_{0}-2 p K_{0} / 3-2\right)} .
$$

The particular case of $p=1(D=0)$ reproduces the original results obtained by van Hemmen [27], and in the case $p=0$ a numerical analysis of Eq. (3.8) provides the second-order phase transition lines for the $F-P$ phase transition in the $T / J-J_{0} / J$ phase space. Thus, the phase diagram shown in Fig. 2 displays second-order phase transition lines (solid lines), tricritical points (black points) and first-order phase transition lines (dotted lines) for several values of the anisotropy parameter $D$ and $p=0$. For $D=-0.01$, or $D \rightarrow 0$, the phase transition $F-P$ is entirely of second-order type. If $D>-0.01$, the system presents tricritical behavior, i.e, the phase transition is second-order in regions with higher temperatures and first-order in regions with lower temperatures, where a tricritical point separates the two regimes. The increase of $|D|$ with $D<0$ leads to first-order phase transition for the region of higher temperatures. Therefore, for $|D| \rightarrow \infty$, the phase transition will be entirely of first-order type. In this case, all spins are under influence of the anisotropic field. We shall notice that there is a competition between $D$ and $J_{0}$ in our model. The increase of $|D|$ for negative values takes the spins remain preferentially in the state with $S=0$, but the growth of $J_{0}$ forces the spins to align themselves parallel. This competition increases the complexity of the system and should induce a first-order phase transition at higher temperatures.

In the case $p \neq 0$, the phase diagram $T / J$ versus $J_{0} / J$ for $D=-0.3$ and several values of $p$, is illustrated in Fig. 3. In the range of $0.14 \geq p \geq 0$, the system presents tricritical behavior. This phase diagram is indicating that for $D=-0.3$ and $p>0.14$ the spin-glass phase disappears, since the second-order phase transition between the ferromagnetic and paramagnetic phases starts at $T / J=0$ and $J_{0} / J=0$, and extends to all other values. Therefore, for any value of $T$ and $J_{0}$ greater than zero there are only ferromagnetic and paramagnetic phases.

For case where $p>p_{c}$ and for all values of $D$, the phase diagram exhibits only second-order phase transition lines and extends down to zero temperature. In the case where $p>p_{c}$ and for any value of $D$, the first-order phase transition lines are

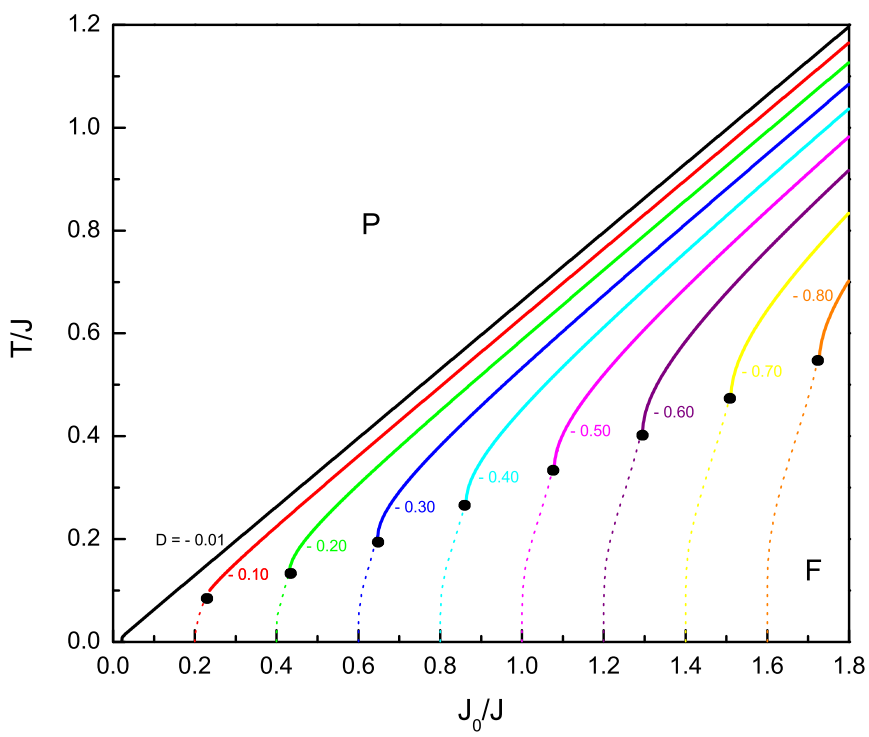

Fig. 2. Phase diagram in the $T / J-J_{0} / J$ plane for the Hemmen model with spin $S=1$ for $p=0$ and different values of $D$, as indicated in the figure. The solid and dotted lines correspond to second- and first-order phase transition lines, respectively. The tricritical points are denoted by a black points. $F$ and $P$, denote the ferromagnetic and paramagnetic phases, respectively. 


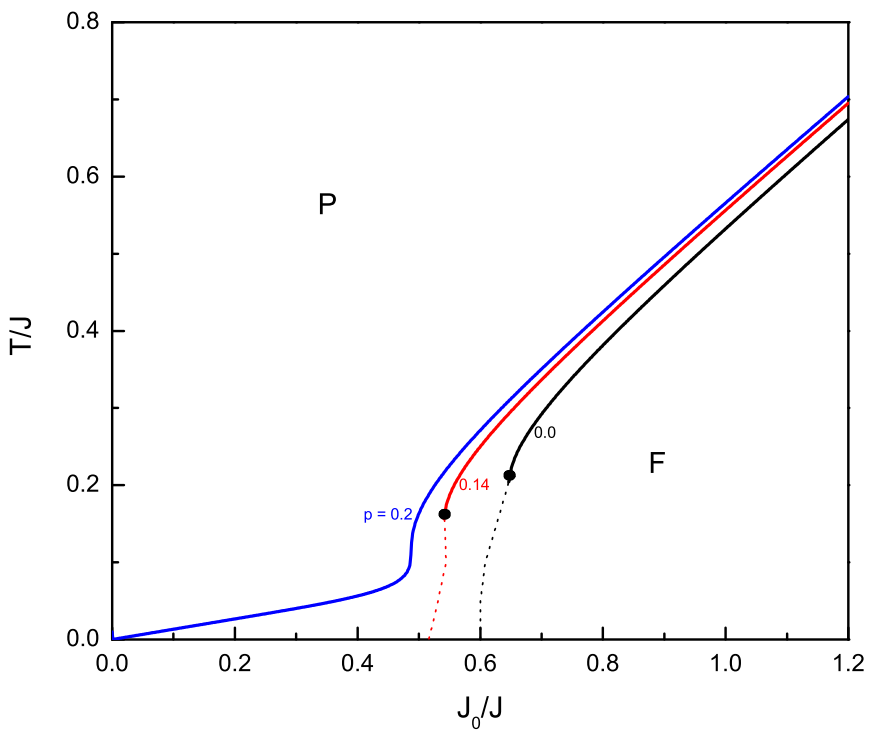

Fig. 3. Phase diagram in the $T / J-J_{0} / J$ plane of the van Hemmen model with spin $S=1$ for $D=-0.30$ and different values of $p$, as indicated in the figure. The solid and dotted lines correspond to the second- and first-order phase transition lines, respectively. The tricritical points are marked by a back points. $F$ and $P$, denote the ferromagnetic and paramagnetic phases, respectively.

suppressed and the second-order phase transition lines extends until the temperature is zero and $J=J_{0}=0$. Fig. 4 exhibits the case $p=0.7$, where the lines begin to merge, meaning that for $p=1$ all the lines merge into a single second-order phase transition line.

Phase transition complete: $F-P-S G$ and mixed phase $(M)$

The analysis of Eqs. (3.1)-(3.3) gives the complete phase diagram of the van Hemmen model with spin $S=1$ and random crystal field. Considering the particular case $p=1$, corresponding to the pure case $D=0$, the phase diagram coincides with the original model of van Hemmen where the vertical axis is multiplied by $2 / 3$. In the ground state $(T=0)$, the expressions of our model reproduce the original result of van Hemmen [27], so that the phase transition $(M-F)$ is found at the same point in the thermodynamic space $J_{0} / J=2 / 3$.

Here, we are mostly interested in the effects of the random crystal field in the phase diagram. Therefore, we considered the cases with $D \neq 0$, having in mind that $D>0$ favors states $S= \pm 1$ and, therefore, in the limit $D \rightarrow \infty$ the system becomes two states $S= \pm 1$, recovering the character the Ising model. On the other hand, if $D<0$, the state with spin $S=0$ is favored and, therefore, when $|D|$ grows for negative values tends to eliminate the mixed phase.

We notice here that the second-order phase transition lines between the $F-P$ (ferromagnetic to paramagnetic), $F-S G$ (ferromagnetic to spin-glass) and $S G-P$ (spin-glass to paramagnetic) phases are obtained by expanding in power series of the order parameters $m_{F}$ in the ferromagnetic phase and $q_{S G}$ in the spin-glass phase, when it becomes zero in a phase transition. On the other hand, in these phase transitions, the first-order phase transition lines are obtained by equating the free energies in each phase and considering the order parameter expressions.

In Fig. 5, we have shown the complete phase diagram of the generalized van Hemmen model for the case $p=0$. This figure displays the results obtained for several values of the crystal field, i.e., $D=-0.10,-0.12,-0.14$ and -0.16 and it shows clearly that the growth of $|D|$ for negative values contributes for the disappearance of the mixed phase. Keeping $p=0$ and increasing the anisotropy for negative values, the effect is to reduce the size of the mixed phase with the growth of the spin-glass phase. When $D$

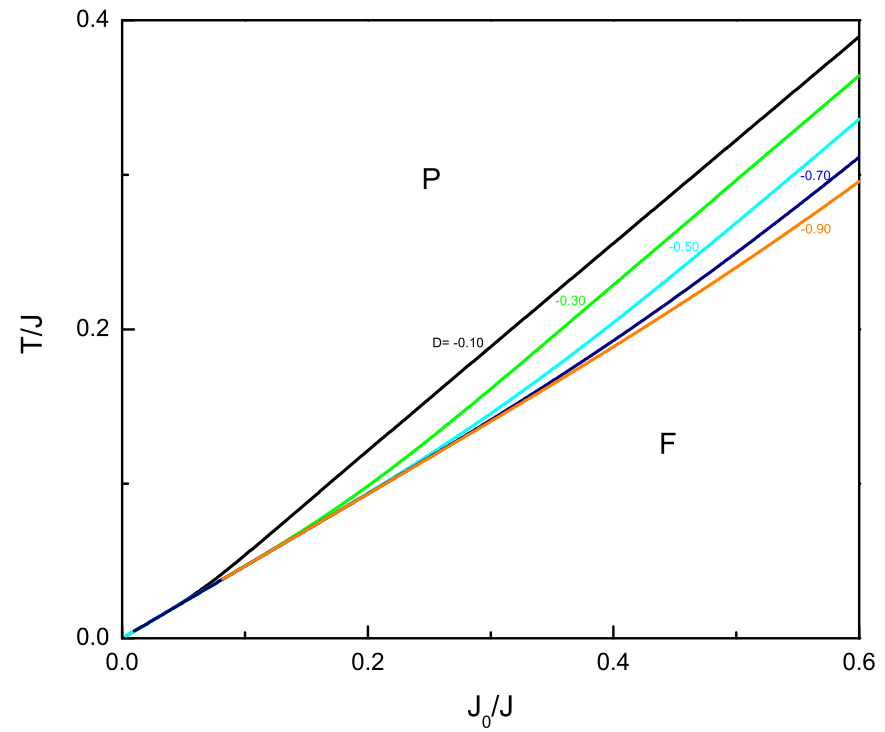

Fig. 4. Phase diagram in the $T / J-D / J$ plane of the van Hemmen model with spin $S=1$ for $p=0.7$ and different values of $D$, as indicated in the figure. The solid lines represent the second-order phase transition lines. $F$ and $P$ denote the ferromagnetic and paramagnetic phases, respectively.

reaches the value $D_{c}=-1 / 6$ the mixed phase disappears completely. This phenomenon can be understood by the fact that negative values of the anisotropy contribute for the state spin $S=0$, therefore, it contributes to the vanishing of the mixed phase. In Fig. 6, we have displayed the values of $p$ and $D$ in which the mixed phase disappears.

Finally, in Fig. 7 we have expressed the phase diagram for several values of $p$ and for a fixed anisotropy $D=-0.2$ in a region where the mixed phase has already disappeared. This diagram shows that increasing the parameter $p$, which corresponds to a dilution in the influence of crystal field, leads the system to increase the size of the ferromagnetic phase, i.e., it exists for smaller values of $J_{0}$.

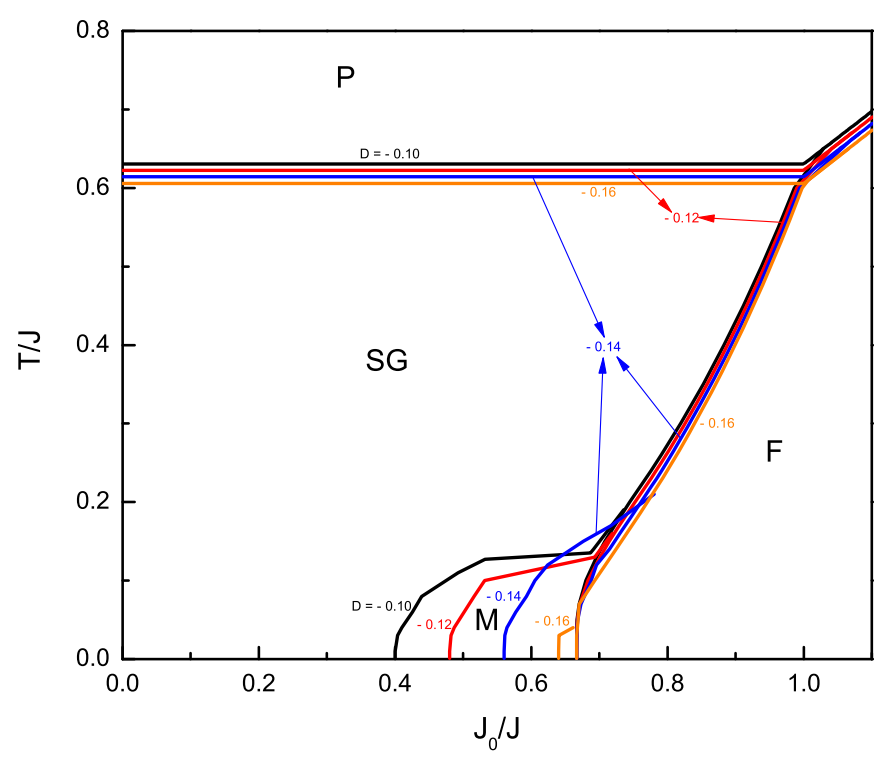

Fig. 5. Complete phase diagram in the $T / J-J_{0} / J$ plane for the van Hemmen model with spin $S=1$ for $p=0$ and several values of $D$, as indicated in the figure. The solid lines represents the second-order phase transition lines. F, $P, S G$ and $M$ denote the ferromagnetic, paramagnetic, spin-glass and mixed phases, respectively. 


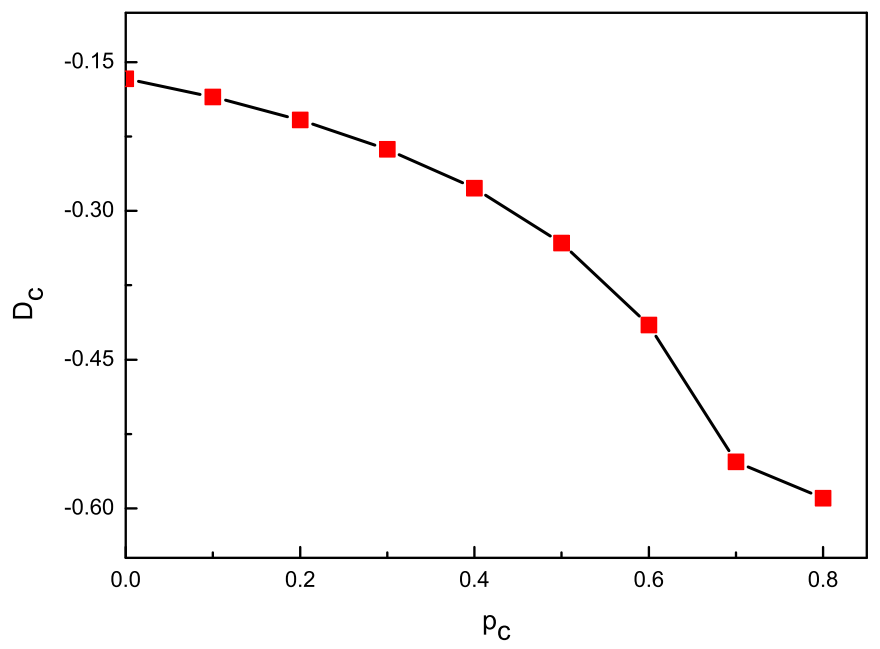

Fig. 6. Phase diagram in the $D_{c}-p_{c}$ plane of the van Hemmen model with spin $S=1$. We have displayed the values of $p$ and $D$ by which the mixed phase disappears.

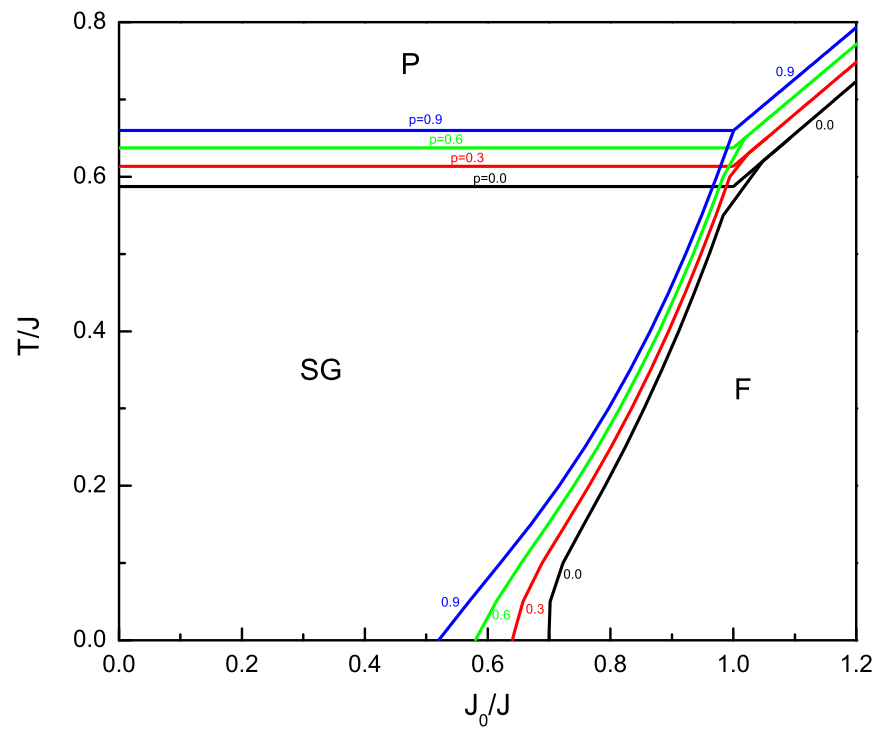

Fig. 7. Phase diagram in the $T / J-J_{0} / J$ plane of the van Hemmen model with spin $S=1$ for $D=-0.2$ and different values of $p$, as indicated in the figure. The solid lines represents the second-order phase transition lines. F, $P$ and $S G$ denote the ferromagnetic, paramagnetic and spin-glass phases, respectively.

\section{Conclusion}

In this paper, we have provided a systematic description of the phase diagram of the van Hemmen model in the presence of random crystal field, for a system with spin $S=1$. Our study were performed for the case of bimodal distributions governing the random interactions (exchange $P(\xi, \eta)$ and random crystal field $P\left(D_{i}\right)$ ), which provides three ordered (spin-glass, ferromagnetic and mixed) phases.

Initially, for $S=1$, the phase transition in the range of $0 \leq p \leq 0.18$, from the spin-glass to paramagnetic phase is of second-order (regions of high temperatures and small values of $|D|$ ) and first-order (regions of low temperature) separated by tricritical points. For $p>0.18$, the phase transition is only of second-order type, and increasing $p$ leads to the behavior found in the canonical van Hemmen model. In case of the phase transitions $F-P$, for $D=-0.01$, i.e., $(D \rightarrow 0)$ the phase transition is entirely of second-order type. If $D>-0.01$, the phase transition is secondorder in regions with higher temperatures, and first-order in regions with lower temperatures, where a tricritical point separates the two regimes. For $|D| \rightarrow \infty$, the phase transition will be entirely first-order.

The complete phase diagram of the generalized van Hemmen model, for the case $p=0$, clearly indicates that the enhancement of anisotropy to negative values contributes for the disappearance of the mixed phase. Keeping $p=0$ and growing the anisotropy parameter, the effect is to reduce the size of the mixed phase with the raise of the spin-glass phase. When $D$ reaches the value $D_{c}=-1 / 6$ the mixed phase disappears completely. We also have provided the values of $p$ and $D$ in which the mixed phase disappears.

\section{Acknowledgments}

The authors acknowledge valuable discussions with L. Craco and J.L.B. de Faria. This work was partially supported by CNPq, FAPEMATInsert here: (Grant No. 685524/2010), FAPEAM and CAPES (Brazilian agencies).

\section{References}

[1] D. Sherrington, Phys. Status Solidi B 251 (2014) 1967.

[2] F.M. Zimmer, M. Schmidt, S.G. Magalhães, Phys. Rev. E 89 (2014) 062117.

[3] M. Schmidt, F.M. Zimmer, S.G. Magalhães, Phys. Scr. 90 (2015) 025809.

[4] S. Fishman, A. Aharony, J. Phys. C 12 (1979) L729.

[5] Y. Imry, S.K. Ma, Phys. Rev. Lett. 35 (1975) 1399.

[6] T. Schneider, E. Pytte, Phys. Rev. B 15 (1977) 1519.

[7] A. Aharony, Phys. Rev. B 18 (1978) 3318.

[8] A.S. Arruda, A. Weizenmann, M. Godoy, D.F. Albuquerque, N.O. Moreno, Physica B 398 (2007) 297.

[9] A.S. Arruda, W. Figueiredo, R.M. Sebastianes, V.K. Saxena, Phys. Rev. B 39 (1989) 4409.

[10] A.S. Arruda, W. Figueiredo, Mod. Phys. Lett. B 11 (1997) 973.

[11] J.R. de Sousa, D.F. de Albuquerque, A.S. de Arruda, Physica A 391 (2012) 3361.

[12] H. Rieger, A.P. Young, J. Phys. A 26 (1993) 5279.

[13] H. Rieger, Phys. Rev. B 18 (1995) 6659.

[14] D. Belanger, in: A.P. Young (Ed.), Spin Glass and Random Fields, World Scientific, Singapore, 1997.

[15] V. Cannella, J.A. Mydosh, Phys. Rev. B 2 (1972) 4220.

[16] Y.J. Uemura, T. Yamazaki, D.R. Harshman, M. Senba, E.J. Ansaldo, Phys. Rev. B 31 (1985) 546.

[17] D. Sherrington, S. Kirkpatrick, Phys. Rev. Lett. 35 (1975) 1792.

[18] R.F. Soares, F.D. Nobre, J.R.L. de Almeida, Phys. Rev. B 50 (1994) 6151.

[19] E. Nogueira Jr., F.D. Nobre, F.A. da Costa, S. Coutinho, Phys. Rev. E 57 (1998) 5079.

[20] T.R. Kirkpatrick, D. Thirumalai, Phys. Rev. B 36 (1987) 5388.

[21] B. Kim, A. Latz, Europhys. Lett. 53 (2001) 660.

[22] K. Binder, A.P. Young, Rev. Mod. Phys. 58 (1986) 801.

[23] J.R. Viana, Y. Nogueira, J.R. de Sousa, Phys. Rev. B 66 (2002) 113307.

[24] P.T. Muzy, A.P. Vieira, S.R. Salinas, Physica A 359 (2006) 469.

[25] V. Jaccarino, in: P.L. Orbach (Ed.), Condensed Matter Physics: The Theodore D. Holstein Symposium, Springer, New York, 1987.

[26] T. Nattermann, J. Vilain, Phase Transit. 11 (1988).

[27] J.L. van Hemmen, Phys. Rev. Lett. 49 (1982) 409.

[28] S.G. Magalhães, F.M. Zimmer, B. Coqblin, J. Phys.: Condens. Matter 23 (2011) 094207.

[29] S.G. Magalhães, F.M. Zimmer, C.V. Morais, Phys. Lett. A 374 (2010) 3554. 